\title{
General Crime Mining Framework for Unstructured Crime Data Prediction
}

\section{Mona Mowafy ${ }^{\# 1}$, A. Rezk ${ }^{\# 2}$, H. M. El-bakry ${ }^{\# 3}$}

\#1 Faculty of Computers and Information, Mansoura University, Egypt, mm_is_85@yahoo.com

\#2 Faculty of Computers and Information, Mansoura University, Egypt, amira_rezk@mans.edu.eg

\#3 Faculty of Computers and Information, Mansoura University, Egypt, helbakry5@yahoo.com

\section{ABSTRACT}

The high volume of unstructured data of crime reports in police stations and the complexity of relationships between these data has made the importance of crime analysis is increasing day after day. This paper proposed a general crime mining framework that integrates the text mining techniques with the crime analysis process to improve the policemen decisions and reduce their efforts. The proposed framework is used in this paper to predict the crime type of the vast amount of unstructured police incident reports, which considered an important part of the criminal behavior analysis. The primary evaluation of the result can predict the crime type with accuracy $85 \%$.

Key words: Crime Analysis Process; Crime Mining Process; Text Analysis; Unstructured Text analysis.

\section{Corresponding Author: Mona Mowafy}

\section{INTRODUCTION}

Criminology is the scientific study of the non-legal portions of crimes, including its characteristics, causes, correction, prevention, the behavior of the criminal involved in the crime, and the social impact of crime [1]. The process of crime analysis is considered an important part of the criminology. In this process all the police reports are reviewed every day to identify patterns and hotspots that provide the law enforcement and intelligencegathering organizations with important information such as who, what, when, where, how and why of emerging crime in the community. This information can be used to solve the complicated crimes, develop effective strategies to prevent or at least reduce the future crimes, find and catch offenders. The main challenges of the crime analysis process [2] are:

- Integration of the collected data: The input data in the crime analysis process is the main element to be used in the analysis process. This data which is obtained from various resources in many formats, it may be in an unstructured form. The challenge is the difficulty of finding the hidden knowledge from large amounts of data.

- Crime patterns: Discovering and predicting the hidden crimes are an important issues in the process of crime analysis. The continuous increasing of the crime rate makes the crime patterns are always changeable and become difficult to be demonstrated and predicated. Therefore the challenge is finding the proper model of detecting crimes, although the continuous changing of the crime patterns. 
- Performance: The issues on performance include the quality, accuracy and processing time. The uncertainty of the results of crime analysis affects the accuracy of the crime detection. In addition, selecting the proper technique affects the processing time. The challenge of performance is developing an effective model to increase the accuracy of crime detection, despite the continued increase in the crime data.

- Visualization: One of the most important issues is helping the text data and analysis results to be more interested and more understood, through visualizing them by creating diagrams, images and animations to provide a suitable summarization for this data. The challenge is how to visualize the data summarizations of the crime analysis result from a huge amount of data.

The increasing size of the unstructured crime data that needs to be stored and analyzed, makes executing the process of crime analysis a time-consuming. Due to the complexity of issues and the investigators, it can take months to solve a crime puzzle. Therefore the crime analysis is an appropriate field for applying the text mining techniques. Using text mining helps the law enforcement agencies to improve the process of crime analysis and overcome its challenges. The gained information from the text mining process can be used to formulate strategies for crime prevention and reduction and improving public safety. The organization of this paper is as follows: section 2 covers the related work of using the text mining technique in the crime analysis, section 3 describes the proposed framework, section 4 illustrates the experimental results of the framework evaluation, and finally section 5 presents the conclusions.

\section{RELATED WORK}

\subsection{Applying the text mining techniques in the crime analysis}

The In the recent decade, using the text mining in the crime analysis field has received a great attention from researchers. This section can be classified according to the used text mining technique as the following:

Shyam [3] has used clustering technique as one of text mining techniques to help police detectives to detect crimes. K-means clustering method has been used with some enhancements to identify crime patterns process. Also Chuan and Jeffery [4] have used clustering technique through the cosine similarity algorithm to compare the police's investigation document and criminal case with the judgments history of court by calculating coefficient of similarity, and according to the highest coefficient, the closest judgment of this type case is found. This can help to generate the draft of indictment for prosecutor. Gangavane, Nikose, and et al. [5] have used a new methodology to present an approach for the evaluation of document clustering of criminal database by using k-means clustering technique. This approach clusters the criminal data basing on the crime's type .

Priyanka and Meghna [6] have used classification technique by presenting a model that based on a fuzzy effective framework to identify the messages that may include number of criminal activities over the web or social network conversation that includes blackmailing, spamming, or cyber the threatening . Also Suman, Madhurima and, et al. [7] have applied the classification technique to identify the irrelevant message or the inclusive crime content in that messages that can be transmitted over the web such emails, chats, tweets, and etc. This approach depends on the fuzzy adaptive approach to identify the positive and negative aspects from the messages. The message will be categorized as the crime or noncrime message in addition to the type of crime in the message. Vijaya Kumar, Balamurugan and, et al. [8] have presented a model for detecting the crime hot spots by a structured classification method. That focus on preprocessing the crime events before the 
DOI: https://dx.doi.org/10.26808/rs.ca.i8v4.02 International Journal of Computer Application (2250-1797)

Issue 8 Volume 4, July-August 2018

mapping. The benefit of this method is that the accurate information of hot spots is more apparent. The classification of crime attribute is the key related to the accuracy of the results. The output of the structured classification creates different type of hotspots based on city crime record.

Sukanya, Kalaikumaran and et al. [9] have used both of clustering and classification techniques to identify the hotspot of criminal activities through classifying the different crimes and grouping the similar types of crimes together. This work helps the police department to increase the security consideration to a particular area to prevent future crimes. Andrey, Bruno and et al. [10] have applied a model based on classification and clustering techniques to a real crime dataset that recorded by police in England and Wales within 1990 to 2011 to classify clustered crimes based on an occurrence frequency during different years. Anshu and Shilpa [11] have used a model that based on clustering and classification techniques to analyze the web data to be able to predict crime trends. They used the k-means as a method of clustering technique methods and then the data is classified to obtain a crime, none and genuine users . Mohammad, Mostafa and et al.[12] have discussed an approach that uses Self-Organizing Map (SOM) and Multi-Layer Perception (MLP) neural network method as clustering and classification methods to cluster and classify crime data through extracting important entities from police narrative reports and enter it into a database.

Raja [13] has applied an information extraction technique through the sentiment analysis on a twitter social media to detect the crime patters by extracting tweets from cities that either be most dangerous or the safest in the United States. Sentiment analysis also applied on tweets to analyze the intensity of the crimes of a particular location. Rabeah and Benjamin [14] have proposed a method to discover criminal networks and extract useful information from a large volume of unstructured text documents obtained from a suspect's machine to help investigators identifying indirect relationships among the members of the identified networks.

Manish and et al. [15] have used the visualization technique by proposing a crime analysis tool that depends on an interactive query interface. This interface can be integrated with the visualization technique to enhance the understanding of the results and patters. The tool is used to extract useful information from a large crime database and find crime hotspots using any text mining technique.

Meshrif [16] has integrated the information extraction, clustering, and visualization techniques by proposing crime profiling system for studding the crime domain in the Arabic language and extracting important information that related to crimes instead of reading entities reports such as (crime type, crime location and nationality of committed persons) to simplify the investigation process for the police investigators. The selforganizing map approach has been used for the process of clustering the crimes based on the predefined attributes and also used in the visualization process that is useful in drawing conclusions and help the user to visualize the summary results of each news report separately.

\subsection{Automatic crime type prediction}

The most of the research studies of automatic crime prediction, applied the classification techniques on structured datasets for predicting the crime type.

Somayeh, Aida, and et al. [17] aimed to classify experimental structured dataset into two categories which are critical and non - critical. They have used five classification algorithms using two ways of feature selection techniques, manually and chi square to determine more accurate classifiers. The experiment results have shown that k-nearest 
neighbor algorithm using chi square achieved the best accuracy. Noora Abdulrahman and Wala Abedalkhader [18] have applied two of data mining techniques - K-nearest neighbor and Naive Bayes to identify and then predict the classification of a crime based on the time and location attributes of the structured dataset of San Francisco. The experimental results have shown that a higher classification accuracy can be obtained by using the Multinomial Naive Bayes.

The problem of missing a large number of discrete attributes of the structured dataset, seriously reduces the classification accuracy. Cuicui, Chunlong, and et al.[19] has proposed a new data filling algorithm, which called GMKNN to improve the classification accuracy for predicting crime types. The proposed algorithm based on combing Mutual Knearest neighbor algorithm with Grey system theory. The proposed GMKNN algorithm increases the classification accuracy to $77,837 \%$. Cuicui, Chunlong, and et al [20] have used three filling algorithms - Maximum class filling algorithm, Roulette algorithm and GBWKNN algorithm to fill the real crime dataset to obtain the complete dataset. Finally the classification accuracy of three algorithms, including C4.5 algorithm, Naive Bayes algorithm, and K-nearest neighbor algorithm, are compared based the completed dataset. The highest accuracy is $66.6939 \%$, which is achieved by combining GBWKNN filling algorithm and K-nearest neighbor classification algorithm.

Rafael , Walkir, and et al. [21] have developed a system that used a weight neural network to classify the unstructured data of the crime reports. The developed system can assist the call center's operator by recommending classification to new reports.

\section{CRIME MINING FRAMEWORK}

Most of the current work is focused on two major directions: (1) Either crime analysis or the text mining trends separately as. (2) Applying the text mining in certain cases of crime analysis as discussed earlier in the related work section. Therefore, this paper proposed framework integrates the text mining technique with the crime analysis process [22] which illustrates a straight line for the interested researchers in their research way. The phases of the proposed crime mining framework as shown in figure 1 are: gathering the unstructured crime data, applying a proper text mining technique, dissemination and feedback.

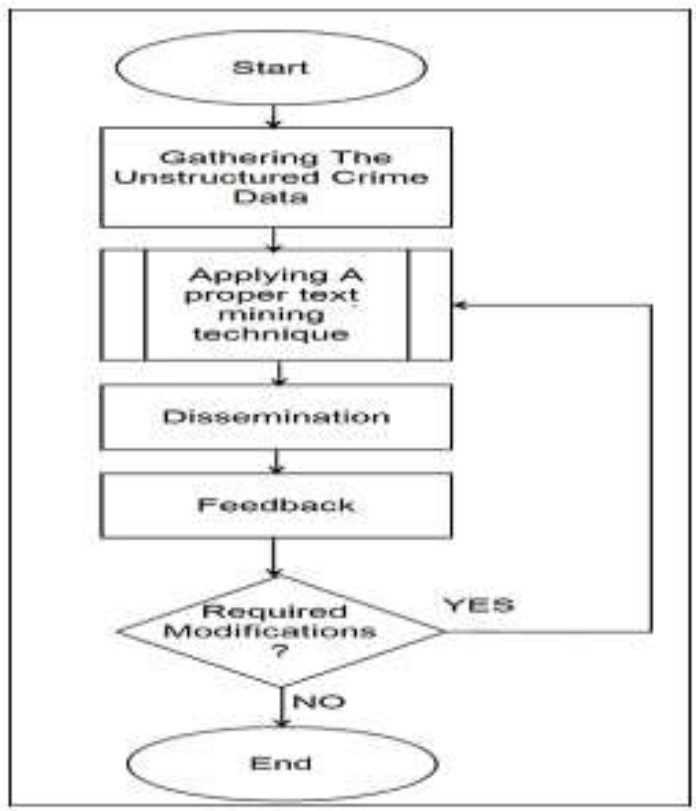

Figure $1:$ architecture of the crime Mining framework 


\subsection{Gathering the unstructured crime data}

This is the first phase of our proposed framework as it is in the standard crime analysis process as shown in figure 2. It is responsible for collecting a lot of documents and data that correctly describe a given task from various resources. The input data type of the proposed framework should be unstructured text documents about the crimes. The unstructured text is the data that isn't organized with a pre-defined manner. This makes it difficult to understand using traditional analysis methods [23].

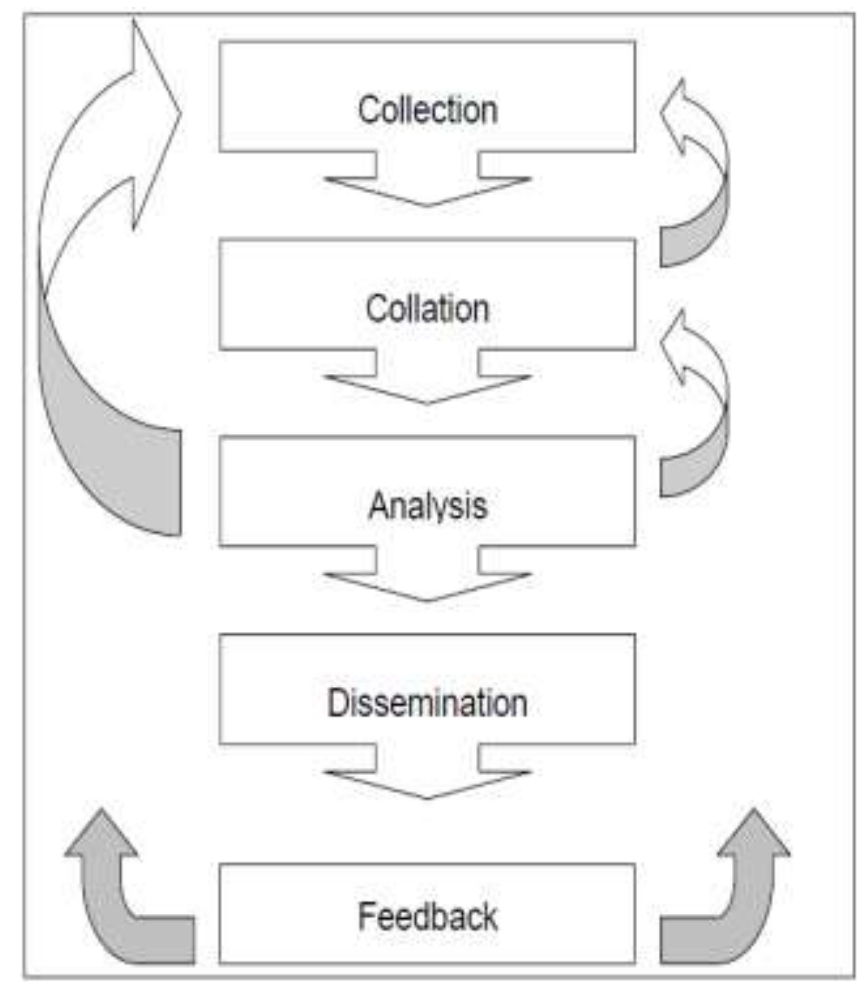

Figure 2: Standard crime analysis process [22]

\subsection{Applying A proper text mining technique}

The phase of applying the text mining in the proposed framework, replaces the data collation and data analysis phases in the standard crime analysis process as in figure [2], that were accomplished manually be system analysts. This phase is an essential component of the crime mining framework. It includes selecting the proper text mining technique that will be applied on the gathered unstructured text data from the previous phase in order to prepare and process them according to the main purpose of the mining process. There are various text mining techniques such as information extraction, classification, clustering, visualization, summarization can be used. The main goal of the phase is examining the prepared data and developing a package of information on crime series, patterns, trends, relationships or connections of the different crimes in different places that should be useful and accurate for dissemination to law enforcement officers.

The authors select the classification model of the unstructured text documents [24] to be applied in this phase as a one of the text mining techniques. The selected model as in figure 3 is responsible for classifying the unstructured data. The selected model uses the Multinomial Naive Bayes as a classification technique, and Term Frequency-Inverse document frequency (TF-IDF) as a vector space model for text extraction for more accurate text classification results. The selected model explains how to build a complete unstructured text classifier. 


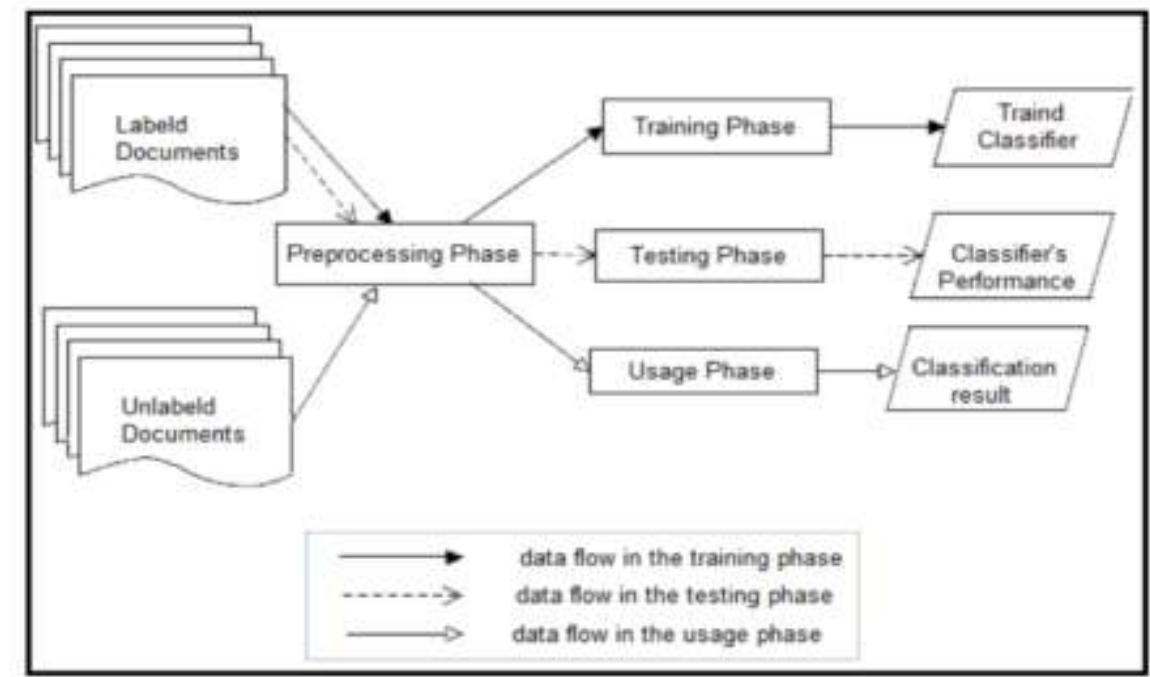

Figure 3: The architecture of the classification model of the unstructured text documents [24]

\subsection{Dissemination}

In this phase, the result of applying the text mining technique in the previous phase will be distributed and communicated to a number of different concerned sides, including law enforcement officers, police management team, and legal organizations.

\subsection{Feedback}

The feedback is the last phase of the crime mining framework. It is responsible for ensuring that crime mining results are meeting user group needs. Feedback from the individuals who uses the analytical reports can lead to more alteration and refinement in the process. Feedback may include the quality of the result, or the usefulness of the result of decision making. According to this returns information that is gained from direct communication with the concerned sides, the selected techniques and methods that used in the phase of applying the text mining technique can be modified and refined.

\section{EXPERIMENT}

Crime type prediction is a decisive part of a criminal analysis. In the most of police stations, it's essential to classify the crimes, according to their types before applying the methods of pattern identification on the crime data. Also the legal organizations aim to divide the crimes into categories for various purposes related to the courts' procedure, such as assigning different types of court to different type of crimes. Crime type prediction is effectively achieved using the classification techniques. Therefore, this experiment based on selecting the classification technique using as a proper text mining technique phase in the proposed framework. The model has been proposed in [24] can be used for classifying the unstructured crime data, according their types based on the details of the police incident reports. This experiment is carried out using a subset of an online dataset that contains police incident reports of the City of Madison [25]. The used dataset consists of the police incident reports of nine major categories of the standard crime types, including battery, burglary, fraud, injury, drug, intoxicated, robbery, theft and weapons violation.

\subsection{Results}

Some performance measures are used to evaluate the proposed framework, which includes accuracy, incorrectly classified, building times, precision, recall, and f1-score as shown in table 1. 
Table 1: Evaluation of the performance framework

\begin{tabular}{|c|c|c|c|c|c|}
\hline $\begin{array}{c}\text { Accuracy } \\
\text { (Correctly } \\
\text { Classified } \\
\text { Instances) }\end{array}$ & $\begin{array}{c}\text { Incorrectly } \\
\text { Classified }\end{array}$ & $\begin{array}{c}\text { Building } \\
\text { Time }\end{array}$ & Precision & Recall & $\begin{array}{c}\text { f1- } \\
\text { score }\end{array}$ \\
\hline $85.3 \%$ & $14.7 \%$ & $0: 00: 05$ & $86 \%$ & $85 \%$ & $85 \%$ \\
\hline
\end{tabular}

The Evaluation of text classification for each crime category is shown in table 2 and the accuracy of each one is presented in figure 4.

Table 2: Performance measures of categories

\begin{tabular}{|l|l|l|l|l|l|}
\hline Category & $\begin{array}{l}\text { Training Set } \\
\text { Total }(\mathbf{6 0 6 7})\end{array}$ & $\begin{array}{l}\text { Testing Set } \\
\text { Total (1517) }\end{array}$ & Precision & Recall & f1-score \\
\hline Battery & 714 & 186 & .84 & .80 & .82 \\
\hline Burglary & 565 & 132 & .95 & .80 & .86 \\
\hline Fraud & 334 & 65 & .98 & .85 & .91 \\
\hline Injury & 236 & 69 & .90 & .83 & .86 \\
\hline Drug & 137 & 21 & .93 & .76 & .78 \\
\hline Intoxicated & 450 & 124 & .89 & .81 & .85 \\
\hline Robbery & 1831 & 512 & .82 & .96 & .88 \\
\hline Theft & 639 & 157 & .79 & .78 & .78 \\
\hline Weapons violation & 1161 & 251 & .88 & .80 & .84 \\
\hline
\end{tabular}

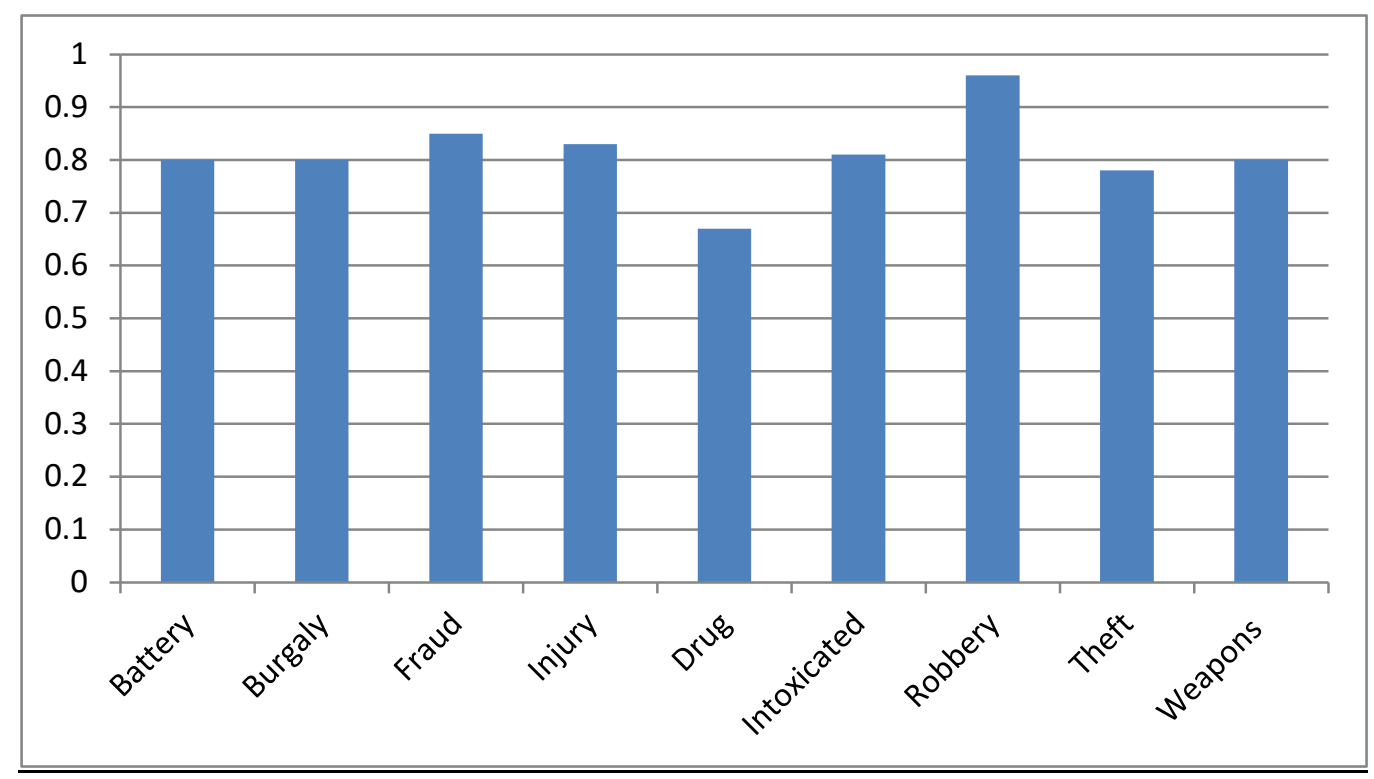

Figure 4: Classification accuracy for each category

The presented results in figure 5, indicate that classification quality of the proposed framework, slightly decreases with the increasing number of testing documents. 


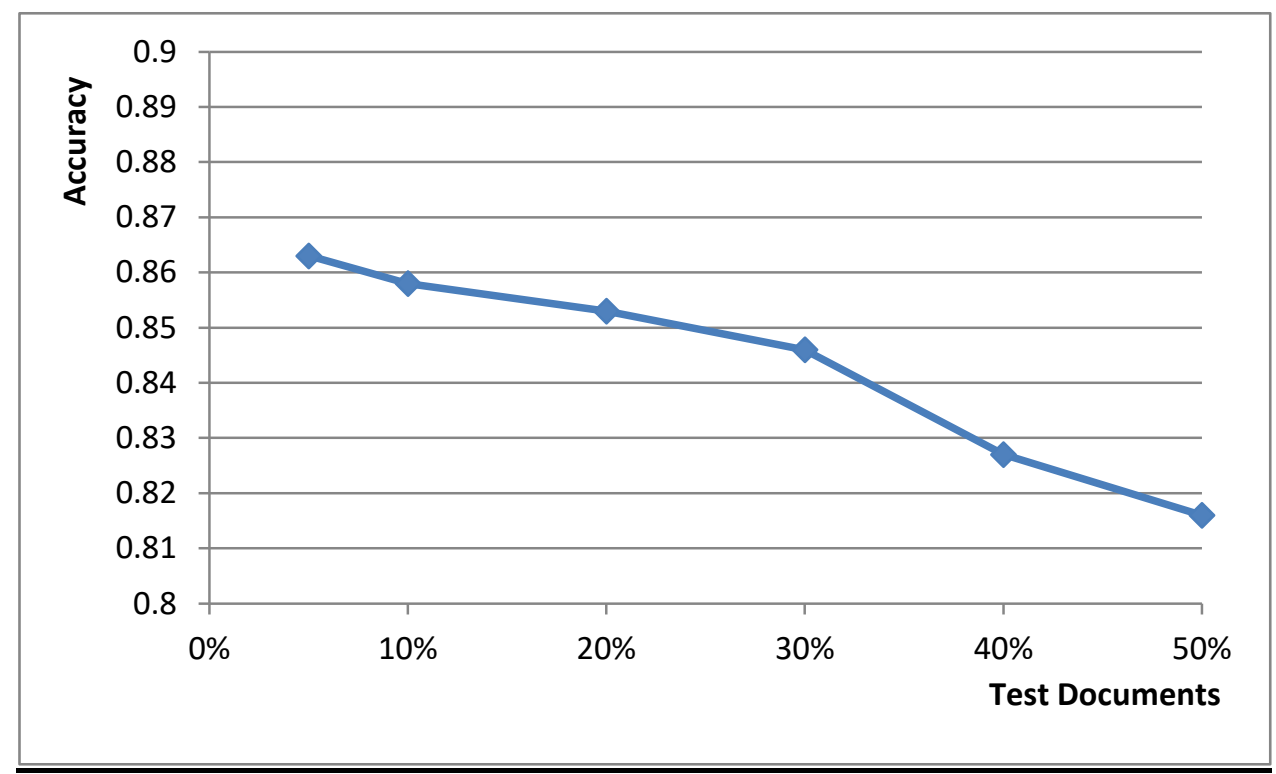

Figure 5: The quality result of classification according to number of testing documents

\section{CONCLUSION}

Criminology is a sensitive domain where text mining techniques play vital role for lawenforcers and crime analysts to facilitate the investigation and help solving unsolved crimes faster. This paper proposed a general framework for a crime mining process that integrates the text mining techniques with the crime analysis process for predicting the crime type through applying text classification for the unstructured data of the police incident reports. Evaluation of the proposed framework with the embedded unstructured classification model that used as the selected technique for the phase of applying the text mining technique, was applied on some of the major categories of crime reports of the dataset of police incident reports of the city of Madison. During testing, the classification performance measures achieved a good result that was very stable and reliable. The proposed framework is extendable for using by further text mining techniques and purposes such as clustering, information extraction, summarization and visualization.

\section{REFERNCES}

I. S. Yamuna, N. Sudha Bhuvaneswari, (2012), Data Mining Techniques to Analyze and Predict Crimes, The International Journal of Engineering And Science (IJES), Volume1, Issue 2, Pages 243-247, ISSN: 2319 - 1813 ISBN: 2319 - 1805

II. Sarpreet Kaur, Williamjeet Singh, Systematic Review of Crime Data Mining, International Journal of Advanced Research in Computer Science, Volume 8, No. 5, May-June 2017.

III. Shyam Varan Nath, (2006), Crime Pattern Detection Using Data Mining, IEEE/WIC/ACM international conference on Web Intelligence and Intelligent Agent Technology, p. 41-44, December 18-22.

IV. Chuan-hsi Chen \&Jeffery Y. P. Chi, (2010), Use Text Mining Approach to Generate the Draft of Indictment for Prosecutor,PACIS 2010 Proceedings. Paper 23.

V. H. N. Gangavane, M. C. Nikose \&P. C. Chavan, (2015), A novel approach for document clustering to criminal identification by using ABK-means algorithm, International Conference on Computer, Communication and Control (IC4), 1-6. 
VI. Priyanka Maan \& Meghna Sharma, (2015), A study on mining approach under cyber crime analysis, International Journal of Emerging Technologies in Computational and Applied Sciences (IJETCAS,) 11 (2), pp. 169-173

VII. Suman, Madhurima, \& Vijay Bhardwaj, (2015), Text mining model to identify criminal activities, International Journal of Applied Research, 1 (7): 391-394

VIII. M. VijayaKumar, P. Balamurugan, Basim Alhadidi \& Hanumanthappa, (2014), Crime Classification Algorithm for Mining Crime Hot Spot and Cold Spot, International Journal of Computing Academic Research (IJCAR), ISSN 2305-9184 Volume 3, Number 2, pp. 58-69

IX. Sukanya. M, T. Kalaikumaran \&Dr. S. Karthik, (2012), Criminals and crime hotspot detection using data mining algorithms: clustering and classification, International Journal of Advanced Research in Computer Engineering \& Technology (IJARCET) Volume 1, Issue 10.

X. Andrey Bogomolov, Bruno Lepri, Jacopo Staiano, Emmanuel Letouze, Nuria Oliver, Fabio Pianesi,\&Alex Pentland, (2015), " Moves on the Street: Classifying Crime Hotspots Using Aggregated Anonymized Data on People Dynamics", Volume 3 Number 3.

XI. Anshu Sharma,\& ShilpaSharma, (2012),"An Intelligent Analysis of Web Crime Data Using Data Mining ", International Journal of Engineering and Innovative Technology (IJEIT) Volume 2, Issue 3.

XII. Mohammad Reza Keyvanpour, Mostafa Javideh, \& Mohammad Reza Ebrahimi, (2011), " Detecting and investigating crime by means of data mining: a general crime matching framework", Procedia Computer Science 3 (2011) 872-880

XIII. Raja Ashok Bolla, (2014), Crime pattern detection using online social media,Masters Theses. , Missouri university of science and technology

XIV. Rabeah Al-Zaidy, Benjamin C. M. Fung, Amr M. Youssef, \&FranciFortin, (2012), Mining Criminal Networks from Unstructured Tex Documents,Digital Investigation, 8 (3-4), pp. 147-160.

XV. B. Chandra and M. P. Gupta, "Crime Data Mining for Indian Police Information System Manish Gupta", Indian Institute of Technology Delhi, Hauz Khas, New Delhi 110016 , India.

XVI. Meshrif Fayad Alruily, (2012), " Using Text Mining to Identify Crime Patterns from Arabic Crime News Report Corpus", PhD Thesis,De Montfort University.

XVII. Somayeh Shojaee, Aida Mustapha, Fatimah Sidi, Marzanah A. Jabar, "A Study on Classification Learning Algorithms to Predict Crime Status", International Journal of Digital Content Technology and its Applicatin, Volume 7, Number 9, May 2013.

XVIII. Noora Abdulrahman and Wala Abedalkhader, "Knn Classifier and Naive Bayes Classifier for Crime Prediction in San Francisco Context ", International Journal of Database Management Systems ( IJDMS ) Vol.9, No.4, June 2017.

XIX. Cuicui Sun, Chunlong Yao, Lan Shen and Xiaoqiang Yu, "Improving Classification Accuracy Using Missing Data Filling Algorithms for the Criminal Dataset", International Journal of Hybrid Information Technology Vol.9, No.4 (2016), pp. 367374.

XX. Cuicui Sun, Chunlong Yao, Xu Li, Xiaoqiang Yu, "Detecting crime types using classification algorithms", BioTechnology: An Indian Journal, Volume 10 Issue 24, 2014, pp. 15451-15457.

XXI. Rafael Adnet Pinho, Walkir A. T. Brito, Claudia L. R. Motta and Priscila Vieira Lima, "Automatic Crime Report Classification through a Weightless Neural Network", 
ESANN 2017 proceedings, European Symposium on Artificial Neural Networks, Computational Intelligence and Machine Learning. Bruges (Belgium), 26-28 April 2017 XXII. Senton, Anna E. , (2009), Crime Analysis: An Examination of Crime Prevention and Reduction Strategies, Master of arts, School of Criminology - Simon Fraser University. XXIII. https://en.wikipedia.org/wiki/Unstructured_data, last accessed on 26/3/2018.

XXIV. Mowafy M, Rezk A, and El-bakry HM," AN Efficient Classification Model For Unstructured Text Document ", American Journal of Computer Science and Information Technology ,2018, Vol.6 No.1:16 XXV. http://data-citymadison.opendata.argis.com, last accessed on 24/2/2018. 